\title{
Mortalidade de pacientes submetidos à cirurgia cardíaca
}

\author{
Mortality of patients undergoing cardiac surgery \\ Mortalidad de pacientes sometidos a cirugía cardíaca
}

Patrícia Farias

ORCID: https://orcid.org/0000-0002-4926-9815 Universidade Federal do Paraná, Brasil E-mail: patrícia.farias@ufpr.br Andrea Moreira Arrué

ORCID: https://orcid.org/0000-0001-5391-324X Universidade Federal do Paraná, Brasil E-mail: andrea.ensp@gmail.com

Tatiana Queiroz Ribeiro de Almeida

ORCID: https://orcid.org/0000-0001-5406-5430 Universidade Federal do Paraná, Brasil

E-mail: tqueirozribeirodealmeida@gmail.com

Leonardo Bigolin Jantsch

ORCID: https://orcid.org/0000-0002-4571-183X

Universidade Federal de Santa Maria, Brasil

E-mail: leo_jantsch@hotmail.com

Angus Wothan Rocha Leites

ORCID: https://orcid.org/0000-0003-4818-7743 Universidade Federal do Paraná, Brasil E-mail: angusroses@gmail.com

Mitzy Tannia Reichembach

ORCID: https://orcid.org/0000-0001-5380-7818 Universidade Federal do Paraná, Brasil E-mail: mitzyr257@gmail.com

\begin{abstract}
Resumo
Objetivo: Avaliar a mortalidade de pacientes pós cirurgia cardíaca em um hospital da região sul. Método: estudo seccional realizado em um banco de dados. Os participantes foram pacientes submetidos à cirurgia cardíaca, no período de julho de 2015 a julho de 2019, totalizando 62 prontuários eletrônicos. O desfecho foi a mortalidade por qualquer causa durante a internação hospitalar com o tipo de cirurgia, histórico de saúde e o perfil do paciente. Resultados: A taxa de mortalidade foi $9,6 \%$, a maioria era do sexo feminino, casado, aposentado, com ensino fundamental completo. A chance de óbito foi maior entre os idosos. A prevalência de complicações pós-operatórias foi $25,8 \%$, sendo mais frequente na cirurgia de válvula cardíaca. As mulheres apresentaram duas vezes mais chance de desenvolver complicações. O tempo de hospitalização também foi 2,6 vezes maior naqueles que apresentaram complicações. O histórico de saúde não foi relevante a nenhum dos desfechos. Conclusões e implicações para a prática: As complicações aumentaram o tempo de internação e a taxa de mortalidade. Avaliar esses fatores possibilita um melhor planejamento da assistência pelo enfermeiro.
\end{abstract}

Palavras-chave: Cirurgia torácica; Mortalidade; Doenças cardiovasculares; Enfermagem.

\begin{abstract}
Objective: To evaluate the mortality of patients after cardiac surgery in a hospital of the southern region. Method: sectional study fulfilled in a database. Participants were patients who underwent cardiac surgery, from July 2015 to July 2019 , totaling 62 electronics medical records. The outcome was mortality from any cause during hospitalization with the type of surgery, health history and the profile of the patient. Results: The mortality rate was $9.6 \%$, most of them female, married, retired, with complete elementary school. The chance of death was higher among the elderly. The prevalence of postoperative complications was $25.8 \%$, being more frequent in heart valve surgery. Women were twice as likely to develop complications. The hospitalization time was also 2,6 times longer in those who had complications. Health history was not relevant to any of the outcomes. Conclusions and implications for the practice: Complications increased the length of hospital internment and the mortality rate. Evaluating these factors allows better planning of care by nurses.
\end{abstract}

Keywords: Thoracic surgery; Mortality; Cardiovascular diseases; Nursing. 


\begin{abstract}
Resumen
Objetivo: Evaluar la mortalidad de los pacientes después de una cirugía cardíaca en un hospital de la región sur. Método: estudio seccional realizado en una base de datos. Los participantes fueron pacientes sometidos a cirugía cardíaca, de julio de 2015 a julio de 2019, totalizando 62 historias clínicas electrónicas. El resultado fue la mortalidad por cualquier causa durante la hospitalización com el tipo de cirugía, antecedentes de salud y perfil del paciente. Resultados: La tasa de mortalidad fue del 9,6\%, la mayoría mujeres, casadas, jubiladas, con primaria completa. La probabilidad de muerte fue mayor entre los ancianos. La prevalencia de complicaciones postoperatorias fue del 25,8\%, siendo más frecuente en la cirugía valvular. Las mujeres tenían el doble de probabilidades de desarrollar complicaciones. El tiempo de hospitalización también fue 2,6 veces mayor en los que presentaron complicaciones. Los antecedentes de salud no fueron relevantes para ninguno de los resultados. Conclusiones e implicaciones para la práctica: Las complicaciones aumentaron la duración de la estancia hospitalaria y la tasa de mortalidad. La evaluación de estos factores permite una mejor planificación de la atención por parte de las enfermeras.
\end{abstract}

Palabras clave: Cirugía Torácica; Mortalidad; Enfermedades Cardiovasculares; Enfermería.

\title{
1. Introdução
}

As doenças cardiovasculares (DCV) constituem a primeira causa de morte no mundo, e no Brasil1 (WHO, 2020, Marinho et al., 2016). Em 2017 ocorreram, em âmbito nacional, 1.312.664 mortes e, dessas 358.882 (27,3\%) foram decorrentes de DCV (Freire et al., 2017). As DCV podem ter tratamento (clínico/medicamentoso) ou cirúrgico, sendo que a segunda opção, reflete em importantes alterações orgânicas e fisiológicas dos pacientes. As cirurgias cardíacas mais comuns são a revascularização do miocárdio (RM) e a correção de doenças valvares, ambas são intervenções complexas e que requerem um tratamento adequado em todas as fases operatórias (Freire et al., 2017).

A progressão das doenças cardiovasculares, bem como possíveis complicações no pós-operatório, estão diretamente relacionadas aos fatores de risco modificáveis e não modificáveis, dentre eles, pode-se destacar: hereditariedade, hipertensão arterial sistêmica (HAS), diabetes mellitus (DM), dislipidemia (DLP), sedentarismo, obesidade e tabagismo (Mello et al., 2019).

Em razão da alta frequência das DCV e, do avanço da medicina, observa-se crescente número de intervenções cirúrgicas torácicas no panorama nacional e internacional. As cirurgias cardíacas mais comuns são a revascularização do miocárdio e a correção de doenças valvares, sendo intervenções complexas e que requerem um tratamento adequado em todas as fases operatórias (Dordetto et al., 2016). Pacientes submetidos a esse tipo de procedimento passam por uma série de exames e testes pré-operatórios e necessitam de cuidados para evitar complicações (Laizo et al., 2010). De acordo com os autores as complicações respiratórias e metabólicas aumentam o tempo de internação em unidade de Terapia Intensiva (UTI).

Portanto, conhecer o perfil clínico dos pacientes bem como os desfechos relacionados a mortalidade e as complicações decorrentes dessa intervenção, podem fornecer informações a equipe, facilitando a elaboração de um plano de cuidados individualizado, melhorando assim a qualidade da assistência e diminuindo o risco de complicações no pós-operatório (Freire et al., 2017). O serviço de saúde e a equipe multidisciplinar tem papel importante na identificação precoce de fatores de risco, para reduzir intercorrências que induzem ao risco de morte de pacientes nesse período.

O objetivo, deste estudo foi avaliar a mortalidade de pacientes submetidos à cirurgia cardíaca em um hospital da região Sul do Brasil.

\section{Metodologia}

Trata-se de estudo seccional analítico, realizado em um sistema informatizado de um hospital, do Estado de Santa Catarina. As cirurgias cardíacas foram implementadas na Instituição em 2015. Foi utilizado o banco de dados proveniente do software Tasy ${ }^{\circledR}$. O prontuário de todos os pacientes hospitalizados é digital e os dados analisados referem-se aos registros eletrônicos de saúde daqueles que foram submetidos à cirurgia cardíaca, no período, de julho de 2015 a julho de 2019.

Os pacientes são submetidos à cirurgia eletiva ou pelo critério de urgência por meio do sistema de saúde conveniado ou particular. Podem ser admitidos no pronto atendimento, no setor de internação ou na enfermaria do hospital, e no momento da 
cirurgia são encaminhados para o bloco cirúrgico. Após a intervenção recebem os cuidados pós-operatórios na UTI.

A UTI possui 10 leitos, equipe multiprofissional composta por cinco enfermeiros, 18 técnicos de enfermagem, um fisioterapeuta e médicos plantonistas. Todos os colaboradores estão distribuídos nos três turnos de trabalho (manhã, tarde e noite).

A pesquisadora principal recebeu o banco de dados, disponibilizado pelo hospital, no mês de setembro de 2019. Os registros eletrônicos dos pacientes submetidos a cirurgia cardíaca, internados na UTI, continham as seguintes variáveis: condições clínicas, individuais e sociodemográficas. As clínicas e individuais eram: idade (variável contínua), sexo (feminino e masculino), data de internação (dia, mês e ano), tipo de cirurgia (revascularização do miocárdio, troca valvar ou implante de marcapasso), comorbidades (hipertensão arterial sistêmica e diabetes mellitus), tabagismo e etilismo (prévio ou atual), complicações (sim e não), desfecho (alta e óbito). As sociodemográficas compreendiam: raça (branca e negra), estado civil (solteiro, casado, separado e viúvo), escolaridade (ensino fundamental, médio e superior) e ocupação (ativo ou aposentado).

Todas as variáveis e dados clínicos, extraídas do banco, foram preenchidas pelos profissionais de saúde da referida Instituição e analisadas pelos pesquisadores. Para análise foi utilizado o software Statistical Package for the Social Sciences (SPSS 22.0) for Windows. Variáveis contínuas foram analisadas pelo teste t de Student para amostras independentes e expressas como média $( \pm)$ desvio-padrão. Variáveis categóricas foram analisadas pelo teste do Qui-Quadrado para comparação de frequência e odds ratio. Amostras pequenas, como raça, idade categórica, estado civil, ocupação, escolaridade utilizou-se o teste exato de Fisher. Foram considerados significativos valores de $\mathrm{p}<0,05$. Destaca-se a ausência de dados em diversos prontuários.

Este estudo obteve aprovação do Comitê de Ética em Pesquisa da Instituição, em 12 de março de 2020, sob o Parecer de $\mathrm{n}^{\circ}$ 3.912.108. Todas as recomendações para pesquisas que envolvem seres humanos foram atendidas em consonância com a Resolução 466/2012, do Conselho Nacional de Saúde (CNS).

\section{Resultados}

Foram avaliados 62 prontuários de todos os pacientes submetidos a cirurgia cardíaca, no período de cinco anos. Avaliouse o desfecho óbito com relação as variáveis individuais, sociodemográficas e clínicas (Tabelas 1 e 2). 
Tabela 1. Desfecho óbito e o perfil dos pacientes submetidos a procedimento cirúrgico torácico. (N=62). Santa Catarina, Brasil, 2021.

\begin{tabular}{|c|c|c|c|c|}
\hline Variáveis & $\begin{array}{l}\text { Óbito } \\
\text { n (\%) }\end{array}$ & $\begin{array}{l}\text { Alta } \\
\text { n (\%) }\end{array}$ & OR (IC95\%) & $p$-valor \\
\hline \multicolumn{5}{|l|}{ Raça/Cor } \\
\hline Branca & $5(8,2)$ & $56(91,8)$ & & $0,097 *$ \\
\hline Negra & $1(100)$ & 0 & & \\
\hline \multicolumn{5}{|l|}{ Estado civil } \\
\hline Casado & $4(9,1)$ & $40(90,9)$ & & \\
\hline Solteiro & 0 & $5(100)$ & & \\
\hline Viúvo & $2(15,4)$ & $11(84,6)$ & & \\
\hline Idade $\left[\sum(\mathrm{DP})\right.$ anos $]$ & $72,50(8,5)$ & $67,54(14,1)$ & & $0,404^{t}$ \\
\hline \multicolumn{5}{|l|}{ Faixa Etária } \\
\hline Adulto & 0 & $14(100)$ & $\begin{array}{c}1,143 \\
(1,027-1,272)\end{array}$ & $0,200 *$ \\
\hline Idoso & $6(12,5)$ & $42(87,5)$ & 1 & \\
\hline \multicolumn{5}{|l|}{ Sexo } \\
\hline Feminino & $4(15,4)$ & $22(84,6)$ & $\begin{array}{c}0,324 \\
(0,055-1,919)\end{array}$ & $0,195^{*}$ \\
\hline Masculino & $2(5,6)$ & $34(94,4)$ & 1 & \\
\hline \multicolumn{5}{|l|}{ Ocupação } \\
\hline Ativo & $1(6,7)$ & $14(93,3)$ & $\begin{array}{c}0,964 \\
(0,080-11,581)\end{array}$ & $0,736^{*}$ \\
\hline Aposentado & $2(6,9)$ & $27(93,1)$ & 1 & \\
\hline \multicolumn{5}{|l|}{ Escolaridade (completa) } \\
\hline Ensino fundamental & $4(15,4)$ & $22(84,6)$ & & \\
\hline Ensino médio & 0 & $13(100)$ & & \\
\hline Ensino superior & 0 & $12(100)$ & & \\
\hline
\end{tabular}

Legenda: *Teste exato de Fisher; ${ }^{\mathrm{t}}$ Teste T de Student. Fonte: Elaborada pelos autores.

A taxa de mortalidade dos pacientes submetidos à cirurgia torácica, no período do estudo, foi de 9,6\% (n=6), a maioria casado, do sexo feminino, aposentado, com ensino fundamental. A ocupação (n=18) e a escolaridade (n=11) tinham dados faltantes. A média de idade dos pacientes que foram a óbito foi maior comparada aqueles que receberam alta ( $\mathrm{p}<0,05)$. 
Tabela 2. Desfecho óbito e alta com o tipo de cirurgia e histórico de saúde. (N=62). Santa Catarina, Brasil, 2021.

\begin{tabular}{|c|c|c|c|c|}
\hline Variáveis & $\begin{array}{l}\text { Óbito } \\
\text { n (\%) }\end{array}$ & $\begin{array}{l}\text { Alta } \\
\text { n (\%) }\end{array}$ & OR (IC95\%) & $p$-valor \\
\hline \multicolumn{5}{|l|}{ Tipo de Cirurgia } \\
\hline Revascularização Miocárdio & $2(8,3)$ & $27(91,7)$ & $\begin{array}{c}0,773 \\
(0,130-4,583)\end{array}$ & $0,572 *$ \\
\hline Valvuloplastia ou Troca Valvar & $4(30,8)$ & $9(69,2)$ & $\begin{array}{c}0,133 \\
(0,027-0,646)\end{array}$ & $0,015^{*}$ \\
\hline Marcapasso & $0(0)$ & $26(100)$ & & $5,14^{*}$ \\
\hline \multicolumn{5}{|l|}{ Histórico de Saúde } \\
\hline Tabagismo & $1(8,3)$ & $11(91,7)$ & $\begin{array}{c}1,200 \\
(0,154-9,345)\end{array}$ & $0,672^{*}$ \\
\hline Hipertensão Arterial Sistêmica & $4(9,5)$ & $38(90,5)$ & $\begin{array}{c}1,050 \\
(0,202-5,262)\end{array}$ & $0,638 * *$ \\
\hline Diabetes Mellitus & $2(15,4)$ & $11(84,6)$ & $\begin{array}{c}0,531 \\
(0,190-2,585)\end{array}$ & $0,369^{*}$ \\
\hline
\end{tabular}

Legenda: *Teste exato de Fisher; ** Teste Qui-Quadrado. Fonte: Elaborada pelos autores.

Alguns pacientes realizaram mais de um tipo de cirurgia $(n=6)$ e tinham mais de uma doença pré-existente (n=5). $O$ tipo de cirurgia com maior número de óbitos foi a valvulopalstia ou troca valvar. A chance do paciente que realizou esse tipo de procedimento ir a óbito foi estatisticamente significativa. Todos que colocaram marcapasso receberam alta. Com relação ao estado de saúde, o paciente ser tabagista, hipertenso ou diabético não interferiu no desfecho óbito. O perfil dos pacientes que desenvolveram complicações em comparação àqueles sem complicações após a intervenção cirúrgica será apresentado na Tabela 3. 
Tabela 3. Desfecho das complicações decorrentes da cirurgia cardíaca, de acordo, com o perfil desses pacientes (N=62). Santa Catarina, Brasil, 2021.

\begin{tabular}{|c|c|c|c|c|}
\hline Variáveis & $\begin{array}{c}\text { Complicação pós-operatória } \\
\text { n }(\%)\end{array}$ & $\begin{array}{c}\text { Sem complicação } \\
\text { n }(\%)\end{array}$ & OR (IC95\%) & $p$-valor \\
\hline \multicolumn{5}{|l|}{ Raça/Cor } \\
\hline Branca & $15(24,6)$ & $46(75,4)$ & & $0,258^{*}$ \\
\hline Negra & $1(100)$ & 0 & & \\
\hline \multicolumn{5}{|l|}{ Estado civil } \\
\hline Casado & $10(22,7)$ & $34(77,3)$ & & \\
\hline Solteiro & $1(20)$ & $4(80)$ & & \\
\hline Viúvo & $5(38,5)$ & $8(61,5)$ & & \\
\hline Idade $\left[\sum(\mathrm{DP})\right.$ anos] & $67,06(15,2)$ & $68,05(13,3)$ & & $0,750^{t}$ \\
\hline \multicolumn{5}{|l|}{ Faixa etária } \\
\hline Adulto & $4(28,6)$ & $10(71,4)$ & $\begin{array}{c}1,200 \\
(0,317-4,541)\end{array}$ & $0,743^{*}$ \\
\hline Idoso & $12(25,0)$ & $36(75,0)$ & 1 & \\
\hline \multicolumn{5}{|l|}{ Sexo } \\
\hline Feminino & $9(34,6)$ & $17(65,4)$ & $\begin{array}{c}2,193 \\
(0,691-6,962)\end{array}$ & $0,178 * *$ \\
\hline Masculino & $7(19,4)$ & $29(80,6)$ & 1 & \\
\hline \multicolumn{5}{|l|}{ Ocupação (n=44) } \\
\hline Ativo & $4(26,7)$ & $11(73,3)$ & 1 & $0,621^{*}$ \\
\hline Aposentado & $8(27,6)$ & $21(72,4)$ & $\begin{array}{c}1,048 \\
(0,257-4,268)\end{array}$ & \\
\hline \multicolumn{5}{|c|}{ Escolaridade $($ completa) $(n=51)$} \\
\hline Ensino fundamental & $7(26,9)$ & $19(73,1)$ & & \\
\hline Ensino médio & $5(38,5)$ & $8(61,5)$ & & \\
\hline Ensino superior & $1(8,3)$ & $11(91,7)$ & & \\
\hline
\end{tabular}

Legenda: *Teste exato de Fisher; $* *$ Teste Qui-Quadrado; ${ }^{\mathrm{t}}$ Teste T de Student. Fonte: Elaborada pelos autores.

A taxa de complicações dos pacientes submetidos à cirurgia torácica, no período do estudo, foi de 25,8\% (n=16). Em relação à raça/cor quase a totalidade $(n=61)$ dos pacientes eram brancos e apenas um paciente negro, $71 \%$ eram casados e $42 \%$ tinham apenas o ensino fundamental completo. A média de idade daqueles que apresentaram complicações foi de 67 anos, muito semelhante ao grupo sem complicações que foi de 68 anos. A ocupação e a escolaridade tinham dados ausentes. A chance de desenvolver complicações não diferiu entre ser adulto ou idoso. No entanto, as mulheres tiveram duas vezes mais chances de desenvolver complicações pós-operatórias do que os homens. 
Tabela 4. Complicações no pós-operatório de cirurgia cardíaca de acordo com tipo de cirurgia e histórico de saúde. (N=62). Santa Catarina, Brasil, 2021

\begin{tabular}{|c|c|c|c|c|}
\hline & $\begin{array}{c}\text { Complicação Pós- } \\
\text { operatória } \\
\text { n (\%) }\end{array}$ & $\begin{array}{c}\text { Sem complicação } \\
\text { n }(\%)\end{array}$ & OR (IC95\%) & $p$-valor \\
\hline \multicolumn{5}{|l|}{ Tipo de Cirurgia } \\
\hline Revascularização do Miocárdio & $6(37,5)$ & $18(39,1)$ & $\begin{array}{c}1,071 \\
(0,332-3,461)\end{array}$ & $0,908 * *$ \\
\hline Valvuloplastia ou Troca Valvar & $7(43,8)$ & $6(13,0)$ & $\begin{array}{c}1,76 \\
(0,969-3,229)\end{array}$ & $0,015^{*}$ \\
\hline Marcapasso & $2(12,5)$ & $24(52,2)$ & $\begin{array}{c}7,636 \\
(1,556-37,467)\end{array}$ & $0,006^{*}$ \\
\hline Dias de UTI $\left[\sum(\mathrm{DP})\right]$ & $8,12(8,09)$ & $3,04(1,98)$ & & $<0,001^{\mathrm{t}}$ \\
\hline \multicolumn{5}{|l|}{ Histórico de Saúde } \\
\hline Tabagismo & $4(25,0)$ & $8(17,4)$ & $\begin{array}{c}1,140 \\
(0,742-1,751)\end{array}$ & $0,371 *$ \\
\hline Hipertensão Arterial Sistêmica & $10(62,5)$ & $39(65,6)$ & $\begin{array}{c}1,371 \\
(0,417-4,513)\end{array}$ & $0,410 * *$ \\
\hline Diabetes Mellitus & $5(31,6)$ & $8(17,4)$ & $\begin{array}{c}1,260 \\
(0,799-1,987)\end{array}$ & $0,204 *$ \\
\hline
\end{tabular}

Legenda: *Teste exato de Fisher; ** Teste Qui-Quadrado; ${ }^{\mathrm{t}}$ Teste T de Student. Fonte: Elaborada pelos autores.

Ressalta-se que 12 pacientes tinham mais de uma comorbidade, totalizando 74 condições de saúde pré-existentes. A chance de o paciente desenvolver ou não alguma complicação após a cirurgia foi semelhante no grupo de tabagistas, hipertenso e diabéticos. A cirurgia com maior número de complicações, pós-operatória, foi a valvuloplastia ou troca valvar. Os pacientes que internaram para realizar o implante de marcapasso, tiveram 7 vezes menos chance de desenvolver complicações. O tempo de hospitalização, na UTI, dos pacientes submetidos a cirurgia cardíaca que apresentaram complicações foi maior que o dobro daqueles sem complicações.

\section{Discussão}

Neste estudo a taxa de mortalidade dos pacientes, foi de 9,6\% e a taxa de complicações de 25,8\%. A taxa de mortalidade hospitalar é indicadora da qualidade da assistência em unidade coronariana (Beccaria et al., 2015), porém, diversas vezes, este dado é utilizado de maneira generalizada, sem que se conheça o perfil da população investigada (Koerich et al., 2016). Existe uma enorme variabilidade de mortalidade nas cirurgias cardíacas, que dependem do hospital, o número de cirurgias e o tipo de procedimento: em cirurgias de revascularização miocárdica, a mortalidade média pode ser de apenas $0,7 \%$ em centros norteamericanos, enquanto a cirurgia de revascularização associada a algum procedimento valvar pode alcançar $20,8 \%$ em alguns centros brasileiros (Monteiro \& Moreira, 2015).

Os resultados deste estudo foram mais baixos quando comparados com resultados de uma pesquisa realizada em um Hospital terciário no sul do país, na qual, a mortalidade observada foi de 14,2\% (Monteiro \& Moreira, 2015).

Complicação pós-operatória é considerada uma segunda doença inesperada, que ocorre até 30 dias após uma cirurgia, alterando o quadro clínico do paciente e essas, continuam presentes, mesmo com os avanços tecnológicos na cirurgia cardíaca e 
investimentos em cuidados perioperatórios, levando a um aumento na mortalidade de pacientes submetidos a esses procedimentos cirúrgicos (Beccaria et al., 2015, Zheng et al., 2016). Este contexto preocupa por impactar na qualidade de vida das populações, além de onerar e sobrecarregar o governo, a sociedade, a família e os indivíduos (Carvalho, Fonseca, Barbosa, Machado, Santos \& Silva, 2015).

No presente estudo a probabilidade de óbito foi maior entre os idosos, dados semelhantes foram observados em estudo que analisou o risco de mortalidade de pacientes submetidos a cirurgia cardíaca a partir do EuroSCORE II (Gutierres et al., 2020).

A maioria dos pacientes submetidos a cirurgias cardiovasculares, no período do estudo, que evoluíram ao óbito eram do gênero feminino com predominância de idosos. A literatura científica indica que os principais fatores de risco para o surgimento de alterações no período pós-operatório de cirurgias cardíacas estão associados à idade do paciente, ao sexo, à hipertensão arterial, ao diabetes, aos níveis elevados de colesterol lipoproteína de baixa densidade (LDL) e baixos de colesterol lipoproteína de alta densidade (HDL), ao tabagismo, à obesidade, ao sedentarismo e à história familiar (Colósimo et al., 2015).

A hipertensão arterial sistêmica (HAS), é considerada o principal fator de risco para doença arterial coronariana e relacionada e ao óbito em pacientes submetidos à revascularização do miocárdio (Beccaria et al., 2015, Albuquerque et al., 2020). Apesar de a HAS representar o antecedente pessoal mais recorrente no presente estudo (79\%), não foi verificada correlação estatística com óbito e complicações pós-operatórias. O histórico de saúde, neste estudo, não foi estatisticamente significativo para o desenvolvimento de complicações entre os grupos de pacientes com hipertensão arterial, diabetes e tabagismo.

Conhecer as complicações pós-operatórias na cirurgia cardíaca direciona a assistência de enfermagem para uma rápida intervenção precoce, favorecendo a terapêutica com consequente redução do tempo de internação e dos custos hospitalares (Ribeiro, 2018).

O Brasil está em segundo lugar no ranking mundial em número de cirurgias cardíacas realizadas anualmente (Barretta et al., 2017). Estudos apontam que a cirurgia de maior prevalência é a revascularização do miocárdio e a valvuloplastia (Santos et al., 2016, Dordetto et al., 2016, Assis et al., 2020). O que vai de encontro com o presente estudo, onde a cirurgia mais realizada foi à revascularização do miocárdio $(38,7 \%)$ e a cirurgia com maior número de complicações, pós-operatória, foi a valvuloplastia ou troca valvar. Na revisão sistemática de estudos randomizados de Kiyose et al. (2019), que avaliou os desfechos de pacientes que receberam aleatoriamente próteses valvares biológicas e mecânicas, mostrou que, apesar de não haver diferenças na mortalidade, há um aumento significativo no risco de nova cirurgia de substituição valvar ao se optar por próteses biológicas. Por outro lado, seu risco de sangramento é menor em comparação ao de próteses mecânicas. Devido à alta prevalência de doença arterial coronariana e com o aumento da expectativa de vida, cada vez mais pacientes, independente do sexo, necessitam de cirurgia de vascularização do miocárdio (Pereira et al., 2017). 


\section{Conclusão e Implicações para prática}

Os pacientes submetidos à cirurgia cardíaca apresentaram maior probabilidade de óbito entre idosos e pós-operatório de válvula cardíaca. Aqueles com complicações tiveram maior tempo de internação em UTI e maior taxa de mortalidade. As mulheres tiveram duas vezes mais chances de desenvolver complicações comparado aos homens. Levando em consideração o papel dos profissionais da saúde como promotores da saúde, acredita-se que os resultados dessa pesquisa possam servir de subsídios para assistência prestada pelo enfermeiro na atenção hospitalar.

A reabilitação de pacientes pós-cirúrgicos, os treinamentos e capacitações das equipes de saúde, a implementação de protocolos assistenciais baseados em diretrizes, a padronização do cuidado, a definição de papéis dentro da equipe multidisciplinar para decisão assertiva baseada em evidência científica, são estratégias que poderão minimizar os riscos de complicações com desfecho de óbito.

O presente trabalho mostrou características importantes de um grupo de pacientes atendidos pelo centro, porém a implantação e o uso contínuo de um banco de dados abrangente com informações dos procedimentos cirúrgicos envolvendo o pré, intra e pós-operatório poderá ampliar estes conhecimentos para trabalhos futuros.

Algumas limitações do estudo devem ser mencionadas tais como o desenho transversal, retrospectivo, com dados secundários, o que limitou as análises de algumas variáveis, principalmente pela ausência de dados nos prontuários. Além do tamanho da amostra decorrente das características locais do cenário de estudo. Finalmente, o fato de ter sido conduzido em um único centro pode limitar a validade externa dos achados no período pós-operatório desses pacientes.

\section{Referências}

Albuquerque, F. L.S ., Sousa, A. E. M., Agostinho, C. N. L. F., Gonçalves, J. R. S., Pimentel, M. I. C., Silva, V. T., Torres, M. A. O., \& Vasconcelos, H. C. A. de. (2020). Obesidade abdominal como fator de risco para doenças cardiovasculares. Braz. J. Hea. Rev., 3(5), 14529-36. 10.34119/bjhrv3n6-066

Assis, C. R., Fortino, C. K., Saraiva, C. A. S., Frohlich, L. F., Silva, R. E., \& Omizzollo, S. (2020). Clinical profile and success in patient extubation after cardiac surgery. J. Physiother. Res, 10 (1), 25-32. 10.17267/2238-2704rpf.v10i1.2619

Barretta, J. C. A., Auda, J. M., Barancelli, M. D. C., \& Antoniolli, D. (2017). Postoperative in cardiac surgery: reflecting about nursing care. J. res.: fundam. care online, 9 (1), pp.259-64. http://dx.doi.org/10.9789/2175-5361.2017.v9i1.259-264

Beccaria, L. M., Cesarino, C. B., Werneck, A. L., Góes, N. C., Santos, K. S., \& Machado, M. N. (2015). Complicações pós-operatórias em pacientes submetidos à cirurgia cardíaca em hospital de ensino. Arq. Ciênc. Saúde, 22 (3), 37-41. 10.17696/2318-3691.22.3.2015.216

Carvalho, C. A., Fonseca, P. C., Barbosa, J. B., Machado, S. P., Santos, A. M., \& Silva, A. A. M. (2015). The association between cardiovascular risk factors and anthropometric obesity indicators in university students in São Luís in the State of Maranhão, Brazil. Cien Saude Colet, 20 (2), 479-90. 10.1590/141381232015202.02342014

Colósimo, F. C., Sousa, A. G., Silva, G. S., Piotto. R. F., \& Pierin, A. M. G. (2015). Arterial hypertension and associated factors in patients submitted to myocardial revascularization. Rev Esc Enferm USP, 49 (2), 200-1. 10.1590/S0080-623420150000200003.

Dordetto, P. R., Pinto, G. C., \& Rosa, T. C. S. C. (2016). Pacientes submetidos à cirurgia cardíaca: caracterização sociodemográfica, perfil clínico-epidemiológico e complicações. Rev. Fac. Ciênc. Méd. Sorocaba, 18 (3), 144-9. 10.5327/Z1984-4840201625868.

Freire, A. K. S., Alves, N. C. C., Santiago, E. J. P., Tavares, A. S., Teixeira, D. S., Carvalho, I. A., Melo M. C. P. de., \& Negro-Dellacqua, M. (2017). Panorama no Brasil das Doenças Cardiovasculares dos últimos quatorze anos na perspectiva da promoção à saúde. Rev. Saúde e Desenvolvimento, 11 (9), 21-44. https://www.uninter.com/revistasaude/index.php/saudeDesenvolvimento/article/view/776

Gutierres, E. D., Rocha, L. P., Tomaschewski-Barlem, J. G., Barlem, E. L. D., Dalmolin, G. L., \& Passos, C. M. (2020). Cirugía cardíaca y riesgo de mortalidad por EUROSCORE II. Research, Society and Development, 9 (4), e66942869. https://doi.org/10.33448/rsd-v9i4.2869

Kiyose, A. T., Suzumura, E. A., Laranjeira, L., Buehler, A. M., Santo, J. A. E., Berwanger, O., Carvalho, A. C. C., Paola, A. A. de, Moises, V. A., \& Cavalcanti, A. B. (2019). Comparison of Biological and Mechanical Prostheses for Heart Valve Surgery: A Systematic Review of Randomized Controlled Trials. Arq. Bras. Cardiol., 112 (3), 292-301. 10.5935/abc.20180272

Koerich, C., Lanzoni, G. M. M., \& Erdmann, A. L. (2016). Factors associated with mortality in patients undergoing coronary artery by-pass grafting. Rev. LatinoAm. Enfermagem, 24, e2748. https://doi.org/10.1590/1518-8345.0708.2748

Laizo, A., Delgado, F. E. F., \& Rocha, G. M. (2010). Complications that increase the time of Hospitalization at ICU of patients submitted to cardiac surgery. Rev Bras Cir Caridiovasc, 25 (2), 166-171. 10.1590/s0102-76382010000200007. 
Research, Society and Development, v. 10, n.5, e12110514610, 2021

(CC BY 4.0) | ISSN 2525-3409 | DOI: http://dx.doi.org/10.33448/rsd-v10i5.14610

Marinho, F. M., Passos, V., Malta, D. C., Barbosa, F. E., \& Abreu, D. M. X. (2016). Burden of disease in Brazil, 1990-2016: a systematic subnational analysis for the Global Burden of Disease Study. Lancet, 392(10149), 760-75. 10.1016/S0140-6736(18)31221-2.

Mello, M. B. de., Silva, C. C. F. da., Real, A. A., \& Albuquerque, I. M. de (2019). Perfil clínico de pacientes submetidos à cirurgia de revascularização do miocárdio e troca valvar em um hospital terciário da região Sul do Brasil. Saúde (Santa Maria), 45(2), 1-11. 10.5902/2236583436298

Monteiro, G. M., \& Moreira, D. M. (2015). Mortalidade em Cirurgias Cardíacas em Hospital Terciário do Sul do Brasil. Int J Cardiovasc Sci., 28 (3), $200-205$. $10.5935 / 2359-4802.20150029$

Pereira, S. E. A., Costa, D., Penido, R., Batista, A. N. S., Ferreira, G. V., Tavares, J. W., Marins, R. B. de., \& Messias, Y. J. (2017). Fatores de risco e complicações de doenças crônicas não transmissíveis. Ciência \& Saúde, 10 (4), 213-219. 10.15448/1983-652X.2017.4.26446

Resolução n.466, de 12 de dezembro de 2012. (2012). Dispões sobre diretrizes e normas regulamentadoras de pesquisas envolvendo seres humanos. Brasília, DF. http://conselho.saude.gov.br/resolucoes/2012/Reso466.pdf

Ribeiro, K. R. A. (2018). Post-operative myocardial revascularization: complications and implications for nursing. J. res.: fundam. care online, 10 (1), pp.242247. http://dx.doi.org/10.9789/2175-5361.2018.v10i1.254-259

Santos, M. B. K., Silveira, C. R., Moraes, M. A. P., \& Souza, E. N. (2016). Desfechos clínicos de pacientes submetidos à cirurgia cardíaca em um hospital do noroeste do Rio Grande do Sul. Rev. Enferm. UFSM, 6 (1), 102-11. 10.5902/2179769216467

World Health Organization [WHO]. (2020, 29 de novembro). Cardiovascular Diseases. https://www.who.int/health-topics/cardiovascular-diseases/\#tab=tab_1

Zheng, Z., Jayaram, R., Jiang. L., Emberson, J., Zhao, Y., Li, Q., Du, J., Guarguagli, S., Hill, M., Chen, Z., Collins R., \& Casadei, B. (2016). Perioperative Rosuvastatin in Cardiac Surgery. N Engl J Med, 374 (18), 1744-1753. 10.1056/NEJMoa1507750. 